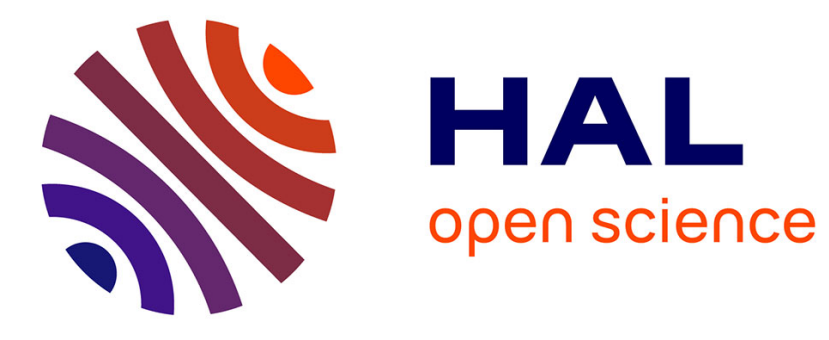

\title{
Hyperspectral image segmentation: the butterfly approach
}

\author{
Nathalie Gorretta, Gilles Rabatel, Jean-Michel Roger, Christophe Fiorio,
} Camille Lelong

\section{- To cite this version:}

Nathalie Gorretta, Gilles Rabatel, Jean-Michel Roger, Christophe Fiorio, Camille Lelong. Hyperspectral image segmentation: the butterfly approach. 1st Workshop on Hyperspectral Image and Signal Processing: Evolution in Remote Sensing (WHISPERS), Aug 2009, Grenoble, France. 10.1109/WHISPERS.2009.5289062 . hal-00468859

\section{HAL Id: hal-00468859 \\ https://hal.science/hal-00468859}

Submitted on 31 Mar 2010

HAL is a multi-disciplinary open access archive for the deposit and dissemination of scientific research documents, whether they are published or not. The documents may come from teaching and research institutions in France or abroad, or from public or private research centers.
L'archive ouverte pluridisciplinaire HAL, est destinée au dépôt et à la diffusion de documents scientifiques de niveau recherche, publiés ou non, émanant des établissements d'enseignement et de recherche français ou étrangers, des laboratoires publics ou privés. 


\section{HYPERSPECTRAL IMAGE SEGMENTATION: THE BUTTERFLY APPROACH}

\author{
Gorretta,N., Roger, JM., Rabatel, G., Bellon-Maurel, V. \\ Cemagref, \\ UMR Itap, \\ Montpellier, France
}

\author{
Fiorio, $C$. \\ Lirmm, \\ CNRS UMR 5506 \\ Montpellier, France
}

\author{
Lelong, $C$. \\ Cirad, \\ UMR TETIS, \\ Montpellier France
}

\begin{abstract}
Few methods are proposed in the litterature for coupling the spectral and the spatial dimension available on hyperspectral images. This paper proposes a generic segmentation scheme named butterfly based on an iterative process and a cross analysis of spectral and spatial information. Indeed, spatial and spatial structures are extracted in spatial and spectral space respectively both taking into account the other one. To apply this layout on hyperspectral imgages, we focus particulary on spatial and spectral structures i.e. topologic concepts and latent variable for the spatial and the spectral space respectively. Moreover, a cooperation scheme with these structures is proposed. Finally, results obtained on real hyperspectral images using this specific implementation of the butterfly approach are presented and discussed.
\end{abstract}

Index Terms - Image segmentation, chemometrics

\section{INTRODUCTION}

Most of the methods devoted to hyperspectral imaging processing conduct data analysis without taking into account spatial information. Pixels are processed individually, as an array of spectral data without any spatial structure. Standard classifications approaches are thus widely used (k-means, fuzzy-c-means, hierarchical classification...). Linear modelling methods such as Partial Least Square analysis (PLS) or non linear approaches like Support Vector Machine (SVM) are also used at different scales (remote sensing or laboratory applications). However, with the development of high resolution sensors, coupling spectral and spatial information when processing complex images appears to be a very relevant approach. Indeed, the integration of the spatial and spectral information in a processing scheme appears important to take advantage of the complementaries that both sources of information can provide [1].

Actually, a few methods for coupling the spectral and the spatial dimensions are proposed in the litterature. The most recent approaches can be broadly classified in two main categories. The first one is related to a direct extension of individual pixel classification methods using just the spectral dimension (k-means, fuzzy-c-means or FCM, SVM). Spatial dimension is integrated as an additionnal classification parameter (Markov fields with local homogeneity constraints [2], SVM with spectral and spatial kernels combination [3], geometrically guided fuzzy C-means [4]...). The second category combines the two fields related to each dimension (spectral and spatial), namely chemometric and image analysis. Various strategies have been attempted. The first one relies on chemometrics methods (Principal Component Analysis or PCA, Independant Component Analysis or ICA, Curvilinear Component Analysis...) to reduce the spectral dimension and then to apply standard image processing techniques on the resulting score images. Another approach is to extend the definition of basic image processing operators to this new dimensionality (extended morphological profiles [5] or watershed segmentation approaches [6] for example).

However, the approaches mentioned above tend to favour only one description either directly or indirectly (spectral or spatial). The purpose of this article is to propose a generic hyperspectral processing approach that strikes a better balance in the treatment of both kinds of information. This method, called butterfly [7], aims to perform an iterative and a cross analysis of data in the spectral and the spatial domains, leading to the segmentation of a hyperspectral image.

This article is organized as follows. Section 2 is devoted to the presentation of the butterfly approach. An implementation of this generic processing scheme is also given. In the following section, experiments performed using the butterfly approach on two hyperspectral images at different resolution scales (proxi-detection and airborne) are described. Results are then presented and discussed.

\section{THE BUTTERFLY APPROACH}

\subsection{Conventions}

Capital bold characters will be used for matrices, e.g. $\mathbf{Z}$; small bold characters for column vectors, e.g. $\mathbf{z}_{i}$ will denote the $i^{\text {th }}$ column of $\mathbf{Z}$; row vectors will be denoted by the transpose notation, e.g. $\mathbf{z}_{j}^{T}$ will denote the $j^{\text {th }}$ row of $\mathbf{Z}$; non bold characters will be used for scalars, e.g. matrix elements $z_{i j}$ or indices $i$.

When the spectral dimension is promoted, a hyperspectral 
image (HSI) is a function $\mathbb{R}^{2}$ to $\mathbb{R}^{p}$ linking a pixel $(x, y)$ to a spectrum $\mathbf{s}(x, y)$, signal vector measured at $p$ wavelength. When the image topology is considered, HSI is a $n \times m$ rectangular set with a partition $\mathcal{P}$ including $k$ connected regions. When the spatial dimension is promoted, a HSI is a 3D matrix noted $\mathbf{Z}_{(n, m, p)}$, with $n$ the number of lines, $m$, the number of columns and $p$ the number of wavelength. For computing reasons, the matrix could be reshaped in a $2 \mathrm{D}$ matrix $(n \times m, p)$ of $n \times m$ spectra built on $p$ wavelengths. Any partition $\mathcal{P}$ is associated with a membership matrix $\mathbf{Q}_{(n \times m, k)}$. Each line of $\mathrm{Q}$, corresponding to one pixel, contains the boolean membership degrees to the $k$ regions.

Let $\mathbf{Z}$ be a HSI with a partition $\mathcal{P}$. Let $\overline{\mathbf{Z}}$ be the mean spectrum of the image and $\overline{\mathbf{Z}}$ the image of the same size as $\mathbf{Z}$ with all pixel values equal to $\overline{\mathbf{Z}}$. Let $\widetilde{\mathbf{Z}}$ be the mean centered matrix, i.e. $\widetilde{\mathbf{Z}}=\mathbf{Z}-\overline{\mathbf{Z}}$. The total inertia matrix of image is defined by: $\mathbf{T}=\widetilde{\mathbf{Z}}^{\mathrm{T}} \widetilde{\mathbf{Z}}$, the between-region inertia matrix is given by $\mathbf{B}=\widetilde{\mathbf{Z}}^{\mathrm{T}} \mathbf{Q}\left(\mathbf{Q}^{\mathrm{T}} \mathbf{Q}\right)^{-1} \mathbf{Q}^{\mathrm{T}} \widetilde{\mathbf{Z}}$ and the within-region inertia matrix is given by $\mathbf{W}=\mathbf{T}-\mathbf{B}$. The Wilks Lambda ([8]) is defined as: $\Lambda\left(\mathbf{Z}, P_{k}\right)=\operatorname{trace}(\mathbf{B}) / \operatorname{trace}(\mathbf{T})$. This index increases from 0 to 1 measuring the gap between regions in the spectral space.

\subsection{Theory}

Given a hyperspectral image $\mathbf{Z}$, the purpose of the butterfly approach is to conduct a segmentation of $\mathbf{Z}$, i.e. a partition $\mathcal{P}$ including $k$ regions $R_{i}$, with $i=1 \ldots k$. To perform this segmentation, a spatial and a spectral structure are identified in the spatial and the spectral space respectively. Next, the available information is reduced accordling to each structure. To ensure cooperation between each space, the butterfly approach is based on an iterative process used to extract, at each round, a spectral structure taking into account a spatial structure and vice-versa. Both extracted structures are thus refined gradually to lead to a final segmentation of the hyperspectral image.

The scheme proposed above is a generic one. To apply it, we have to focus on specific spatial and spectral structures and to define cooperation between them. In this article, we will focus solely on particular notions of topology for the spatial space i.e. the notions of connectivity and adjacency and the building of latent variables for the spectral space. In addition, to ensure collaboration, concepts of regions and false colors or score images (spatial space) are transfered to concepts of classes and latent variables (spectral space) respectively. The above is summarized in figure 1 .

Thus, one "butterfly" round is made up of two steps:

1. Extraction of a spatial structure (topology) based on a spectral structure,

2. Extraction of a spectral structure (latent variables) based on a spatial structure.

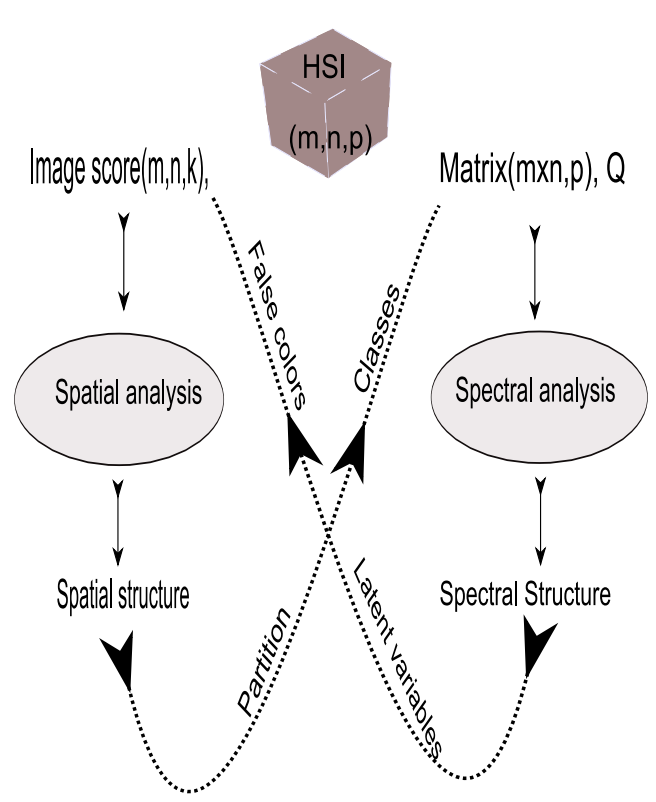

Fig. 1. The butterfly approach

These steps are processed in a successive, iterative and interdependent way.

The first step deals with the use of commonly used image processing tools (region segmentation algorithms) on a limited number of score images. This makes it relatively easy to process. To carry out the second step, chemometric tools are used to reveal a subspace (latent variables) which enables us to characterize the data according to the membership matrix $\mathbf{Q}$ and the optimization of a criterion based on the between $(\mathbf{B})$ or the within inertia $(\mathbf{W})$ of the data. The choice of this criterion is dependent on the region segmentation strategy used i.e. either a top down approach (splitting) or a bottomup approach (merging). According to the theorical principles of these approaches, the used criterion will be the maximisation of $\mathbf{B}$ and $\mathbf{W}$ for the bottom-up and top down strategy respectively.

\subsection{Implementation}

In this paper, the butterfly approach is implemented using a split and merge strategy. First, we over-segment the image by a serie of split based on spectral and spatial features following the butterfly approach. Secondly, we merge back the regions, in order to get the number of expected regions or to satisfy a stop criterion.

Each split operation is conducted by projecting the image data on $k_{s}$ latent variable determined by the diagonalisation of the within inertia matrix (the first $k_{s}$ eigenvectors). Next, split 
of all exiting region in $p$ regions are tested and the one that maximizes the Wilks Lambda $\left(\Lambda\left(\mathbf{Z}, P_{k}\right)\right)$ criterion is chosen. Besides, to ensure a progressive segmentation process and a good complementarity between extrated structures (spectral, spatial) at each round, $p$ is adjusted to 2 .

Each merge operation is conducted by projecting the image data on $k_{m}$ latent variables determined by the diagonalisation of the between inertial matrix (the first $k_{m}$ eigenvectors). Next, merge of all adjacent regions are tested and the one that maximises the Wilks Lambda is chosen.

\section{MATERIAL AND METHODS}

In order to evaluate the performance of the proposed approach, two data sets were used:

- The first one is a DAIS (Digital Airborne Imaging Spectrometer) hyperspectral image data in the reflective and thermal wavelength ranges $(496-20000 \mathrm{~nm}$ with 80 bands) from the city of Pavia in Italy. To reduce processing time, this image was reduced to obtain a final image size $(n=140) \times(m=160) \times(p=80)$. Black and white image (band 50) is given Figure 2-a.

- The second one was acquired in field (plant identification) with a Hyspex VNIR-1600 hyperspectra system (Norsk Elecktro Optikk) in 160 narrow bands (400-980 $\mathrm{nm}$ with $3.6 \mathrm{~nm}$ spectral resolution). To reduce processing time, this image was binned to obtain a final image size $(n=120) \times(m=150) \times(p=160)$. Color image is given Figure 2-b.

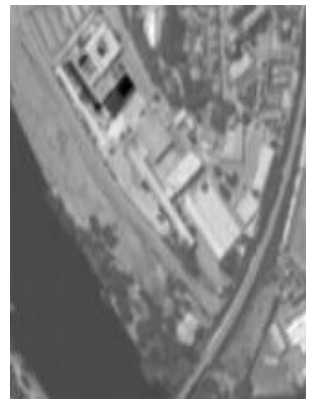

(a) Image DAIS (band 50)

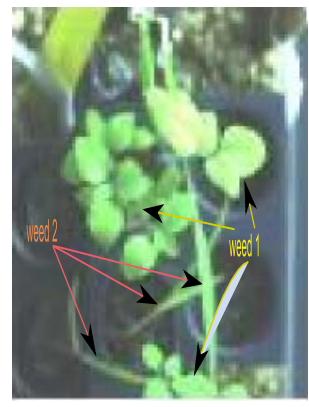

(b) Image Hyspex
Fig. 2. Hyperspectral images

For the split phase, we used the normalized cut algorithm as proposed by Shi [9]. A weighted graph of the image score is then constructed by taking each pixel as a node, and connecting each pair of pixels by an edge. The edge weight $w(i, j)$ between pixel $i$ and $j$ is defined as the product of an intensity similarity term and spatial proximity term and measures the likelihood fo pixel $i$ and $j$ belonging to the same image region:

$$
w(i, j)=\left\{\begin{array}{l}
e^{\frac{-\left\|X_{i}-X_{j}\right\|^{2}}{\sigma_{x}}} e^{\frac{-\left\|I_{i}-I_{j}\right\|^{2}}{\sigma_{I}}} \text { if }\left\|X_{i}-X_{j}\right\|<R, \\
0 \text { otherwise }
\end{array}\right.
$$

where, $X_{i}$ and $I_{i}$ denote pixel location and intensity (image score), $R$ a radius, $\sigma_{x}$ and $\sigma_{I}$ regularisation parameters.

For both images $k_{s}$ and $k_{m}$ are adjusted to 1 and $99,99 \%$ respectively. In fact, for the split phase, one latent variable is enough to decide if two regions are not similar. Even more, two regions that are different on one latent variable will be necessary on more. On the contrary, for the merge phase, to be sure that two regions are similar, it is necessary to look at many latent variables. Indeed, $k_{m}$ is adjusted to explain a large amount of explained variance.

\section{RESULTS}

Figures 3-a and b show segmentation results obtained on the DAIS image for the split and merge phase respectively. Regularisation $\left(\sigma_{I}\right.$ and $\left.\sigma_{x}\right)$ and $R$ parameters were fixed to 1.0, 15 and 20. According to the number of urban structures included in the image, split and merge phase was stopped as soons as 15 and 12 regions have been found respectively. That corresponds to 14 split rounds and 3 merge rounds.

On this image, obtained segmentation allows to extract a majority of urban structures and more particulary buildings. However, the split phase leads to the segmentation of the inland waterways and the road is not wholly detected. These errors could be explained by the poor quality of some spectral bands which do have not been left out for the treatment.

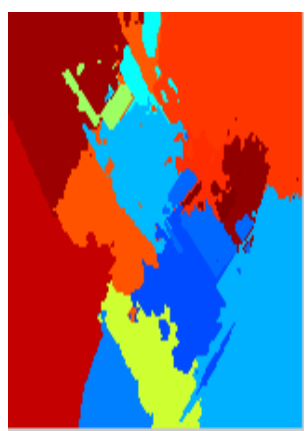

(a) End of split stage

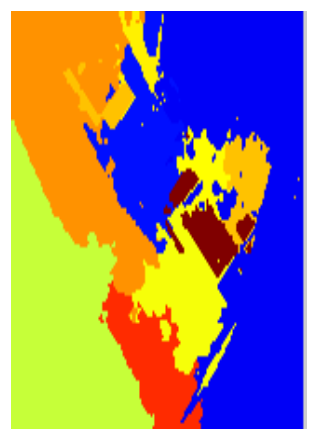

(b) End of merge stage
Fig. 3. Results on DAIS image

Figures 4-a and b show segmentation results obtained on the hyspex image for the split and merge phase respectively. Regularized $\left(\sigma_{I}\right.$ and $\sigma_{x}$ ) and $R$ parameters were fixed to 0.8 , 
20 and 15. Split and merge phase was stopped as soons as 10 and 5 regions have been found respectively. That corresponds to 9 split rounds and 5 merge rounds. On this image, segmentation results are particulary relevants. Indeed, vegetation and background are correctly segmented.

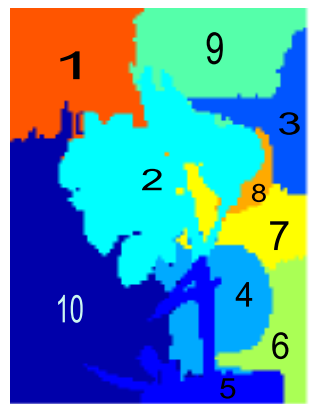

(a) End of split stage (b) End of merge stage

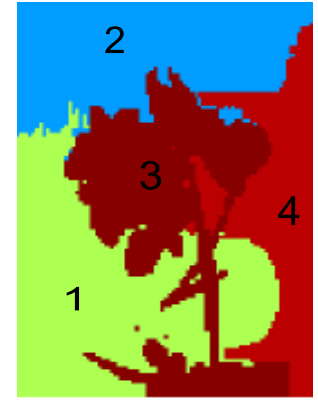

Fig. 4. Results on Hyspex image

\section{CONCLUSION}

In this paper, a generic approach to segment hyperspectral images has been proposed. This method called butterfly is based on an iterative process and a cross analysis of spectral and spatial structures. To demonstrate the relevance of this layout , we have focused on particular spectral and spatial structures i.e. topologic (connectivity) and latent variables concepts for the spatial and the spectral space respectively. In this case, cooperation between extracted structures was made using heterogeneity concepts. In addition, we have implemented this approach by using a split and merge segmentation process and a normalized cuts segmentation algorithm. Results obtained on in field and airbone hyperspectral images are promising. They must be confirmed by additional tests on other images and with ground truth for a quantitative evaluation.

However, as mentionned above, butterfly approach is a generic one. Indeed, a perspective of this work lies in the determination of other spectral and spatial structures and colloboration processes to address a wide variety of image segmentation problems.

\section{ACKNOWLEDGMENT}

The authors would like to thank Dr. Paolo Gamba from the University of Pavia, Italy, for the DAIS data set.

\section{REFERENCES}

[1] Antonio Plaza, Jon Atli Benediktsson, Joseph W. Boardman, Jason Brazile, Lorenzo Bruzzone, Gustavo CampsValls, Jocelyn Chanussot, Mathieu Fauvel, Paolo Gamba,
Anthony Gualtieri, Mattia Marconcini, James C. Tilton, and Giovanna Trianni, "Recent advances in techniques for hyperspectral image processing," Remote Sensing of Environment, vol. In Press, pp. -, 2009.

[2] O. Prony, X. Descombes, and J. Zerubia, "Classification des images satellitaires hyperspectrales en zone rurale et priurbaine," Rapport de recherche INRIA 4008, INRIA, septembre 2000.

[3] G. Camps-Valls, L. Gomez-Chova, J. Munoz-Mari, J. Vila-Frances, and J. Calpe-Maravilla, "Composite kernels for hyperspectral image classification," Geoscience and Remote Sensing Letters, vol. 3, no. 1, pp. 93 - 97, 2006.

[4] J. C. Noordam and W.H.A.M. Van den. Broek, "Multivariate image segmentation based on geometrically guided fuzzy c-means clustering," Journal of Chemometrics, vol. 16, pp. 1-11, 2002.

[5] J.A. Benediktsson, J.A. Palmason, and J Sveinsson, "Classification of hyperspectral data from urban areas based on extended morphological profiles," IEEE Transactions on Geoscience and Remote Sensing, vol. 43, no. 3, pp. 480-491, 2005.

[6] G. Noyel, J. Angulo, and D. Jeulin, "Morphological segmentation of hyperspectral images," Image Analysis and Stereology, vol. 26, pp. 101-109, 2007.

[7] N. Gorretta, J-M Roger, C. Fiorio, V. Bellon-Maurel, G. Rabatel, and C. Lelong, "Proposition d'une stratégie de segmentation d'images hyperspectrales," "Revue de traitement du signal", vol. "In Press", pp. "-", 2009.

[8] S Wilks, Multidimensional scatter, Standford Press, 1960.

[9] J. Shi and J. Malik, "Normalized cuts and image segmentation," in IEEE Conference Computer Vision and Pattern Recognition(CVPR), Puerto Rico, 1997. 